\title{
Two-face(s): ionized and neutral gas winds in the local Universe
}

\author{
A. Concas ${ }^{1, \star}$, P. Popesso $^{1}$, M. Brusa ${ }^{2,3}$, V. Mainieri ${ }^{4}$, and D. Thomas ${ }^{5}$
}

\author{
1 Excellence Cluster Universe, Boltzmannstr. 2, 85748 Garching, Germany \\ e-mail: a.concas@mrao.cam.ac.uk \\ 2 Dipartimento di Fisica e Astronomia, Universitá degli Studi di Bologna, Via Piero Gobetti 93/2, 40139 Bologna, Italy \\ 3 INAF-Osservatorio Astronomico di Bologna, Via Piero Gobetti, 93/3, 40139 Bologna, Italy \\ ${ }_{5}^{4}$ European Southern Observatory, Karl-Schwarzschild-str. 2, 85748 Garching, Germany \\ 5 Institute of Cosmology and Gravitation, University of Portsmouth, Dennis Sciama Building, Burnaby Road, \\ Portsmouth PO1 3FX, UK
}

Received 23 October 2017 / Accepted 24 November 2018

\begin{abstract}
We present a comprehensive study of the Na I 15890,5895 (Na I D) resonant lines in the Sloan Digital Sky Survey (SDSS, DR7) spectroscopic sample to look for neutral gas outflows in the local galaxies. Individual galaxy spectra are stacked in bins of stellar mass $\left(M_{\star}\right)$ and star formation rate (SFR) to investigate the dependence of galactic wind occurrence and velocity as a function of the galaxy position in the SFR- $M_{\star}$ plane. While in most cases the interstellar medium (ISM) absorption and emission lines are at the galaxy systemic velocity, in massive galaxies $\left(M_{\star}>5 \times M_{\odot}\right)$ at the high SFR tail (SFR $>10-12 M_{\odot} \mathrm{yr}^{-1}$ ) we find evidence of a significant blue-shifted $\mathrm{Na} I \mathrm{D}$ absorption, which we interpret as evidence of neutral outflowing gas. The occurrence of the blue-shifted absorption in the stacked spectra does not depend on the contribution of the nuclear activity as it is observed at the same significance for purely star-forming (SF) galaxies, active galactic nuclei (AGN), and composite systems at fixed SFR. We confirm, instead, for all classes of objects a clear dependence on the galaxy disc inclination: the blueshift is the largest and the Na I D equivalent width the smallest for face-on galaxies, while the absorption feature is at the systemic velocity for edge-on systems. This indicates that the neutral outflow is mostly perpendicular or biconical with respect to the galactic disc. We also compare the kinematics of the neutral gas with the ionized gas phase as traced by the [OIII] $\lambda 5007, \mathrm{H} \alpha$, [NII] 26548 , and [NII] 6584 emission lines in the same galaxy spectra. Differently for the neutral gas phase, all the emission lines show evidence of perturbed kinematics only in galaxies with a significant level of nuclear activity, and they are independent of the galactic disc inclination. This would suggest that, while neutral winds originate from the galactic disc and are powered by SF feedback, ionized outflows are instead due to AGN feedback originating from the black hole accretion disc. In both the neutral and ionized gas phases, the observed wind velocities (of the order of 100-200 kms ${ }^{-1}$ ) suggest that the outflowing gas remains bound to the galaxy with no definitive effect on the gas reservoir.
\end{abstract}

Key words. ISM: jets and outflows - ISM: lines and bands - galaxies: general - galaxies: active - galaxies: ISM galaxies: star formation

\section{Introduction}

Galaxies are not equally efficient in converting baryons into stars. It is now well established that the highest efficiency is reached by central galaxies of $10^{12-12.5} M_{\odot}$ dark matter halos, like our own Milky Way, and drops down quickly on both sides of this threshold towards lower and higher halo masses (e.g. Madau et al. 1996; Baldry et al. 2008; Conroy \& Wechsler 2009; Guo et al. 2010; Moster et al. 2010, 2013; Behroozi et al. 2010, 2013). According to the current theoretical framework, outflows of different natures are the most viable way to expel the gas from a system and so regulate the star formation process through the gas availability (e.g. Di Matteo et al. 2005; De Lucia et al. 2006; Croton 2006; Hopkins et al. 2006, 2014; Bower et al. 2006; Henriques et al. 2017). In low-mass halos $\left(<10^{12-12.5} M_{\odot}\right)$ a combination of supernovae explosions and stellar winds in actively star-forming (SF) regions, is sufficient to sweep the gas away (see Chevalier 1977; Murray et al. 2005 and Hopkins et al. 2014, respectively, for energy-driven outflows, momentum-driven outflows, and effect of multiple stel-

\footnotetext{
* Current affiliation: Kavli Institute for Cosmology, University of Cambridge, Madingley Road, Cambridge CB3 OHA, UK
}

lar feedback in cosmological simulations). In massive halos $\left(>10^{12} M_{\odot}\right)$, instead, the deeper potential well requires more energetic sources to expel the gas from the central system. Powerful feedback from active galactic nuclei (AGN) is believed to provide an effective mechanism in this respect (Di Matteo et al. 2005; De Lucia et al. 2006; Croton 2006; Hopkins et al. 2006, 2014; Bower et al. 2006; Henriques et al. 2017) because the energy generated by the growth of the supermassive central black hole (BH) exceeds the binding energy of the gas by a large factor (see Fabian 2012 and King \& Pounds 2015).

Despite the ability of these models in predicting a large variety of evidence, a clear observational consensus regarding (i) the efficiency of the different feedback as a function of stellar or dark matter halo mass and (ii) their actual effect in regulating the star formation process is still lacking. Over the past decade, a lot of effort has been made to provide observational evidence of the existence and effect of the outflows. Star formation driven outflows are nearly ubiquitously observed in highly active SF galaxies at all cosmic epochs (see Veilleux et al. 2005 and Erb 2015 for a comprehensive overview), usually associated with energetic starburst phenomena (e.g. Heckman et al. 1990; Pettini et al. 2000; Rupke et al. 2005a,b; Martin 2005, 2006; Hill \& Zakamska 2014). Even so, 
their role in regulating the availability of gas is still largely debated (e.g. Martin et al. 2012a; Rubin et al. 2014; Steidel et al. 2010). Similarly, energetic outflows driven by AGN feedback are observed at low (e.g. Feruglio et al. 2010; Villar-Martín et al. 2011; Rupke \& Veilleux 2011, 2013a; Mullaney et al. 2013; Rodríguez Zaurín et al. 2013; Concas et al. 2017) and high redshift (e.g. Maiolino et al. 2012; Brusa et al. 2015; Perna et al. 2015; Harrison et al. 2016; Zakamska et al. 2016) with either positive (enhancement) or negative (suppression) effects on the galaxy star formation activity (Cresci et al. 2015; Maiolino et al. 2017; Cresci \& Maiolino 2018).

It is now well established that galactic winds are complex multi-phase phenomena, observed in neutral, ionized, and molecular gas phase in SF galaxies (e.g. Heckman et al. 1990; Veilleux et al. 2005; Rupke et al. 2005a,b; Bordoloi et al. 2014; Erb 2015) and in AGN dominated objects (e.g. Feruglio et al. 2010, 2015; Villar-Martín et al. 2011; Harrison et al. 2014; Genzel et al. 2014; Brusa et al. 2015, 2016; Concas et al. 2017). However, there is still no general framework able to explain the relations between the different wind phases and their respective statistical incidence (see also Cicone et al. 2018). This is likely because most observational evidence to date has been based on relatively small samples of peculiar objects such as luminous and ultra-luminous infrared galaxies (LIRGs and ULIRGs; e.g. Heckman et al. 1990; Cazzoli et al. 2014, 2016) and massive AGN (e.g. Greene \& Ho 2005; Mullaney et al. 2013; Förster Schreiber et al. 2018) leading to strong selection biases. The low statistics often leads to inconclusive or contradictory results (see e.g. Rupke et al. 2017 and Perna et al. 2017b). Only few attempts have been made to study the incidence and the role of outflows in the bulk of the galaxy population. Concas et al. (2017) based on Sloan Digital Sky Survey (SDSS; Abazajian 2009) stacked SDSS optical spectra, show that outflows of the ionized gas phase are taking place with a much higher incidence and velocity shift in galaxies with a strong AGN contribution and in particular at high stellar masses $\left(M_{\star}\right.$, see also Cicone et al. 2016 for a similar study). Chen et al. (2010) used the high signal-to-noise ratio $(\mathrm{S} / \mathrm{N})$ of SDSS stacked spectra to study the incidence of galactic outflows in the neutral gas phase through the $\mathrm{Na} I \mathrm{D}$ absorption feature in the population of SF galaxies. They find a strong dependence on outflow incidence as a function of the disc inclination, which favours a biconical or perpendicular outflow geometry. However, no systematic study has been conducted so far to study the relative incidence and properties of different gas phase outflows in the bulk of the galaxy population.

In this paper, we conduct this systematic study by extending the analysis of the ionized gas phase of Concas et al. (2017) to the neutral gas phase. For this purpose we use the spectroscopic sample of the SDSS at $z<0.3$ with a stacking analysis defined in Concas et al. (2017). As a tracer of the neutral gas phase we use the Na I 15890,5895 (Na I D) resonant lines, which has been largely used to detect the wind signature of blue-shifted absorbing material in front of the continuum source (e.g. Heckman et al. 2000; Rupke et al. 2005a,b,c, 2017; Martin 2005; Rupke \& Veilleux 2015; Cazzoli et al. 2016). Our goal is to characterize the kinematics of the neutral outflows in the bulk of galaxy population at low redshift to (i) investigate how they are related to the host galaxy properties such as star formation rate (SFR), $M_{\star}$, and galaxy geometry; (ii) identify the main triggering mechanism: star formation, AGN, or a mixed contribution of the two effects; and (iii) explore whether there is a difference between this cool neutral gas and the warmer ISM traced by the

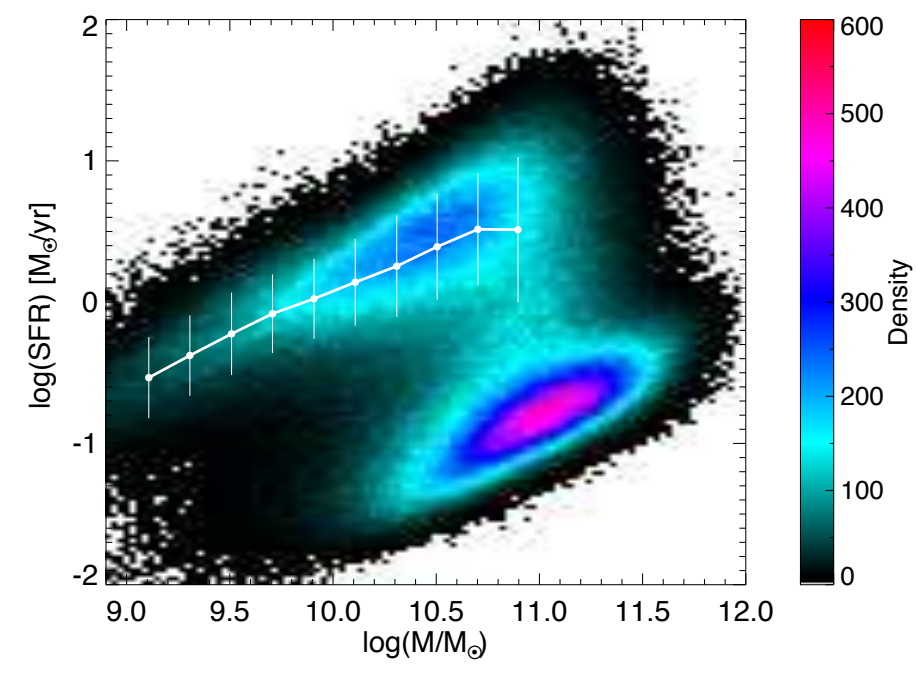

Fig. 1. SFR vs. $M_{\star}$ plane for our DR7 SDSS galaxies, colour-coded by the object density as indicated on the right. The white line shows the position of the main sequence of SF galaxies, computed as the mode and the dispersion of the SFR distribution in stellar mass bins following the Renzini \& Peng (2015).

[OIII] $\lambda 5007, \mathrm{H} \alpha,[\mathrm{NII}] \lambda 6548$, and [NII] $\lambda 6584$ emission lines in the same galaxies.

This paper is organized as follows. We describe our sample selection and physical properties in Sect. 2. The details of the method of creating stacked spectra and measuring the $\mathrm{Na}$ I $\mathrm{D}$ line profile are described in Sect. 3. We present and discuss the main results in Sects. 4 and 5, respectively. Finally, we summarize our findings in Sect. 6. Throughout this paper, we adopt the following $\Lambda \mathrm{CDM}$ cosmology with $H_{0}=70 \mathrm{~km} \mathrm{~s}^{-1} \mathrm{Mpc}^{-1}$, $\Omega_{\mathrm{M}}=0.3$, and $\Omega_{\Lambda}=0.7$.

\section{Data}

The data set used in the present study is based on the SDSS (York et al. 2000) spectroscopic catalogue, data release 7 (DR7; Abazajian 2009). In particular, we analysed objects drawn from the Main Galaxy Sample (MGS; Strauss et al. 2002) which have Petrosian magnitude $r<17.77$ and redshift distribution spanning $0.005<z<0.30$. The spectra are obtained with $3^{\prime \prime}$ diameter fibres that cover a wavelength range from $3800<\AA<9200$, with an instrumental resolution of $R \equiv \lambda / \delta \lambda \sim 1850-2200$ and a mean dispersion of $69 \mathrm{~km} \mathrm{~s}^{-1}$ pixel $^{-1}$. See the SDSS web page ${ }^{1}$ for more exhaustive details concerning the DR7 spectra.

Throughout the paper we use the $M_{\star}$ and SFR estimates of the the MPA-JHU catalogue, which provides derived galaxy properties for more than 800000 galaxies $^{2}$. Briefly, the stellar masses are estimated by fitting the broad-band optical photometry (Kauffmann et al. 2003) and the SFR measurements are based on the Brinchmann et al. (2004) procedure. The $\mathrm{H} \alpha$ emission line luminosity is used to determine the SFRs for the SF galaxies and the D4000-SFR relation (e.g. Kauffmann et al. 2003) for all galaxies that have emission lines contaminated by AGN activity or that are undetected. This leads to a SFR estimate for all objects. All SFR measures are corrected for the fibre aperture as described by Salim et al. (2007). We restrict the sample to stellar mass $\log \left(M / M_{\odot}\right) \geq 9.0$ to limit the incompleteness

\footnotetext{
http://www.sdss.org/dr7/

http://WwW.mpa-garching.mpg.de/SDSS/DR7/
} 
in the low-mass regime. The galaxy sample is shown in the SFRstellar mass plane in Fig. 1, and it contains 600 000 objects (see Concas et al. 2017, hereafter C17, for further details on the sample description).

The MPA-JHU catalogue also includes the flux values of several emission lines for each galaxy. In order to distinguish normal SF from AGN dominated galaxies we classify galaxies in the Baldwin et al. (1981; hereafter BPT) diagnostic diagram on the basis of the line ratios: [OIII] $\lambda 5007 / \mathrm{H} \beta$ and $[\mathrm{NII}] \lambda 6584 / \mathrm{H} \alpha$. The use of the [NII] $\lambda 6584 / \mathrm{H} \alpha$, in particular, allows us to accurately discriminate between the ionization due to the presence of photons originating from SF and AGN activity. As reported by previous studies, this emission line ratio is more sensitive to the presence of low-level AGN than other diagnostics such as $[\mathrm{SII}] / \mathrm{H} \alpha$ and $[\mathrm{OI}] / \mathrm{H} \alpha$ (see e.g. Kewley et al. 2006; Schawinski et al. 2010). Following the Stasinska et al. (2006) classification, we define subsamples on the basis of the prevalence of different photoionization processes: SF, SF-AGN, AGN-SF, LINERs, and TYPE 2, respectively 20.6\%, 7.4\%, $11.2 \%, 5.6 \%$, and $1.7 \%$ of the total sample. In this paper the LINERs and TYPE 2 galaxies are classified together as AGN galaxies. All galaxies with no emission lines or very weak ones $(S / N<4$, about $53 \%$ of the total sample) are not classifiable using the BPT diagram and we call these objects "un-Class". As already mentioned in the Introduction, the main goal of this study is to investigate and quantify the evidence of neutral gas outflows in the bulk of galaxy populations in the local universe; therefore, the inclusion of such non-emitter galaxies is mandatory in the following analysis. However, we checked that our main results are not affected by the inclusion of the un-Class galaxies.

ISM Na I D in galaxy spectra. In this work we focus on the $\mathrm{Na}$ I doublet at $\lambda \lambda 5890,5896 \AA^{3}$ resonant lines in the restframe optical galaxy spectra. It is now generally recognized that more than one process causes the formation of this transition in a galaxy spectrum, including the absorption and re-emission of stellar radiation by the ISM and the absorption of radiation produced in the stellar interiors by the most external gas layers (stellar contribution). It is clear that in order to identify any signature of outflow in the ISM component this radiation must be isolated through the removal of the stellar component. This can be done by fitting the stellar continuum so as to derive the stellar contamination of the $\mathrm{Na} I \mathrm{D}$ feature. This analysis requires very high $\mathrm{S} / \mathrm{N}$ spectra, first to properly fit the stellar continuum and also to obtain a high $\mathrm{S} / \mathrm{N}$ residual ISM component in order to derive the gas kinematics. Only the stacking analysis of the SDSS spectra allows us to reach such high S/N. However, this poses a problem. The ISM component can be in emission or absorption, with an incidence strongly dependent on the dust obscuration (Chen et al. 2010). The emission and absorption in the stacking of a spectral feature can easily cancel each other out and so lead to very biased results. Thus, before proceeding with the stacking procedure described in the next section, we first verify the incidence of emission and absorption of the $\mathrm{Na}$ I D interstellar component in the SFR- $M_{\star}$ plane.

\footnotetext{
3 The transition that gives rise to the doublet is from the ground-state configuration $3 \mathrm{~s}$ to the energy levels of the $3 \mathrm{p}$ configuration composed of two states with total angular momentum $j=3 / 2$ and $j=1 / 2$ (spinorbit effect). The continuum photons emitted by the stellar component are absorbed by the $\mathrm{Na}$ I atoms (at temperatures $T<1000 \mathrm{~K}$ ) and are reemitted when the electrons in the $3 p$ configuration decay spontaneously to the ground state.
}



Fig. 2. SFR vs. $M_{\star}$ plane for our DR7 SDSS galaxies, colour-coded by the percentage of objects showing a $\mathrm{Na}$ I D excess $\left(\mathrm{NaD}_{\text {obs }}>\mathrm{NaD}_{\text {mod }}\right)$ in each bin, from the Na I D measurements of the MPA-JHU catalogue; only object with $S / N>2$ in the single observed Na I D Lick measurements were included. The Na I D excess objects dominate the high- $M_{\star}$ region, while the number of the $\mathrm{Na} I \mathrm{D}$ deficiency $\left(\mathrm{NaD}_{\text {obs }}<\mathrm{NaD}_{\text {mod }}\right)$ objects increases at low stellar masses. The white line shows the mode and dispersion of the main sequence (see Fig. 1).

This test is done by using the information contained in the MPA-JHU catalogue ${ }^{4}$, which provides a measure of the $\mathrm{Na}$ I D line strength parameterized by the Na I D Lick index (as defined in Worthey et al. 1994). The catalogue includes a measure of the Na I D Lick index due to the stellar component $\left(\mathrm{NaD}_{\text {stellar_mod }}\right)$, derived by fitting the continuum as described in Tremonti et al. (2004), together with a measure of the observed $\mathrm{Na}$ I D Lick index $\mathrm{NaD}_{\text {obs }}$, which is given by the combined ISM and stellar contribution. $\mathrm{A} \mathrm{NaD}_{\text {obs }}>\mathrm{NaD}_{\text {stellar_mod }}$ (hereafter Na I D excess) implies an extra ISM absorption component, while a $\mathrm{NaD}_{\text {obs }}<\mathrm{NaD}_{\text {stellar_mod }}$ (hereafter $\mathrm{Na}$ I D deficiency) points to an ISM emission component. The distribution of $\mathrm{Na}$ I D excess and deficiency is not perfectly Gaussian, as outlined by Chen et al. (2010), but it exhibits a long tail on the positive side (excess). We investigate the incidence of ISM emission and absorption in the SFR- $M_{\star}$ plane only for the SDSS subsample, where $\mathrm{NaD}_{\text {obs }}$ is observed at $S / N>2$. As shown in Fig. 2, the $\mathrm{Na} I \mathrm{D}$ excess and deficiency are not homogeneously distributed in the plane, but they exhibit a bimodal distribution as a function of the galaxy stellar mass. The deficiency dominates $(80 \%)$ at low stellar masses, and the excess dominates at the high-mass end. Only in the transition region at $10^{10-10.5} M_{\odot}$ are emission and absorption mixed together in similar percentages. This is consistent with recent high-redshift galaxy studies of the ISM FeII and MgII ISM component in the rest-frame near-UV, tracing the same gas phase (see Finley et al. 2017; Feltre et al. 2018). The observed bimodal distribution of the Na I D deficiency and excess allows us to perform the stacking analysis in the SFR- $M_{\star}$ plane with the caveat that in the central region, at $M_{\star}=10^{10-10.5} M_{\odot}$, the result might be biased.

\footnotetext{
4 https://wwwmpa.mpa-garching.mpg.de/SDSS/DR7/SDSS_ indx.html
} 

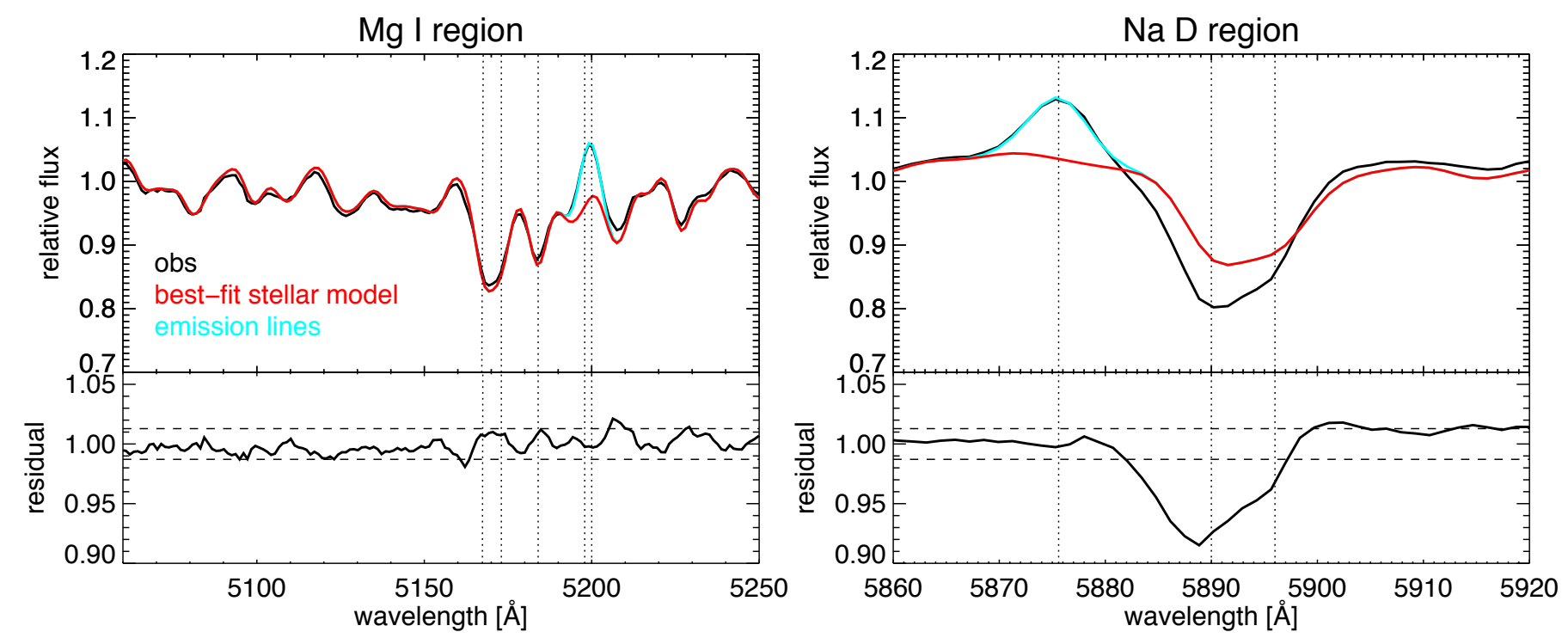

Fig. 3. Example of our continuum fit and subtraction performed for the stacked spectrum with $\log \left(\mathrm{SFR} / M_{\odot} \mathrm{yr}^{-1}\right)=1.4$ and $\log \left(M_{\star} / M_{\odot}\right)=10.9$ in the Mg I and Na I D regions (left and right panel, respectively). The observed stacked spectrum is shown with a solid black line, the best-fit stellar continuum model in red and stellar emission lines in cyan. The dotted lines indicate the stellar Mg I triplet at $\lambda \lambda 5167,5173,5184 \AA$ and the $[\mathrm{NI}] \lambda 5198,5200 \AA$ emission lines (left panel), and the He I $\lambda 5876$ emission and the Na I doublet $\lambda \lambda 5890,5896$ (right panel). The bottom panels show the residual spectra (solid black line) and the residual variations (dashed lines). The Mg I region is reproduced well by the stellar fit with residuals lower than the mean scatter. The $\mathrm{Na}$ I D fit is instead affected by significant residuals, revealing a blue-shifted extra absorption component.

\section{Method}

In this section, we describe how we fitted and removed the stellar absorption contribution and ionized emission lines and how we measured the properties of the ISM Na I D resonant line in our stacked spectra.

\subsection{Stacked spectra and stellar continuum fit}

In order to improve the $\mathrm{S} / \mathrm{N}$ of our single spectra and, at the same time, to connect the Na I D feature to the galaxy properties (SFR, $M_{\star}$, and main ionization source), we stacked together the optical spectra of the C17 sample in bins of SFR and $M_{\star}$ $\left(\Delta \log \left(M / M_{\odot}\right)=0.2\right.$ and $\left.\Delta \log (\mathrm{SFR})=0.2 \mathrm{dex}\right)$. Following the approach of $\mathrm{C} 17$, we obtained 148 galaxy stacked spectra in the C17 sample all over the SFR- $M_{\star}$ plane (see the fine grid in Fig. 1 in $\mathrm{C} 17)$.

We used a combination of two publicly available codes: pPXF Cappellari \& Emsellem (2004) to fit the stellar continuum, and GANDALF Sarzi et al. (2006) to fit the nebular emission lines. As in $\mathrm{C} 17$ we used the $\mathrm{BC} 03$ stellar library (Bruzual \& Charlot 2003) to produce a model of the stellar continuum that matches the observed line-free continuum in the wavelength range $3800<\lambda<6900 \AA$. Our templates included simple stellar population with ages $0.01 \leq t \leq$ $14 \mathrm{Gyr}$, four different metallicities, $Z / Z_{\odot}=0.2,0.4,1,2.5$ and assumed a Chabrier (2003) initial mass function (IMF). Any interstellar medium emission and absorption lines, including $\mathrm{Na}$ I D, were ignored in the continuum fit. The emission lines were separately fitted with GANDALF. The two contributions, continuum+emission lines, were then subtracted from the observed spectrum to isolate the ISM component. Figure 3 shows an example of the stellar continuum (red curve) and emission line (cyan curve) fit versus the observed spectrum (black curve) in two regions, the $\mathrm{Mg}$ I (left panel) and $\mathrm{Na} \mathrm{I} \mathrm{D}$ (right panel). In both plots the bottom panels show the residual after the subtraction of continuum+emission lines. While the $\mathrm{Mg}$ I triplet at $\lambda 5167,5173,5185$, due only to stellar atmosphere, is very well fitted (it is known that $\mathrm{EW}(\mathrm{NaD})_{\text {star }}=$ $0.75 \times \mathrm{EW}(\mathrm{MgIb})_{\text {star }}$ Heckman et al. 2000, see also Rupke et al. 2002, 2005a; Villar Martín et al. 2014), the Na I D region exhibits the expected extra absorption of the ISM component.

In the right panel, in particular, we highlight the importance of removing the He I $\lambda 5876$ emission line in order to properly isolate the Na I D ISM contribution. This emission is due to the presence of energetic photons able to photoionize the He atoms in the HII regions in SF galaxies (see also Chen et al. 2010). For these galaxy spectra the He I line is fit with a single Gaussian component and then removed from our spectra. We check that a multi-Gaussian fit is not required for this emission line. This residual spectrum (bottom right panel in Fig. 3) is finally used for any analysis of $\mathrm{Na} \mathrm{D}$ line features.

The error on the residual spectrum is obtained through bootstrap re-sampling methods shown in $\mathrm{C} 17$.

\subsection{Measuring the ISM Na I D absorption line profiles}

In our stacked spectra, the velocity structure of the $\mathrm{Na}$ I D line is generally complicated; this resonant line shows absorption, emission, or a combination of the two contributions across the physical parameters of the galaxies (as also found in the SF galaxy spectra showed in Chen et al. 2010 and in the nearby quasars in Rupke \& Veilleux 2015; Rupke et al. 2017). To correctly quantify the centroid velocity and velocity dispersion of the $\mathrm{Na} I \mathrm{D}$ resonant line, a careful treatment of the absorption and emission lines is mandatory. Each stacked spectrum is fit with three distinct models using the IDL MPFIT fitting code.

Model 1. The first model, the one - free - doublet model, consists of a pair of Gaussians with a single kinematic component (as in previous works, e.g. Davis et al. 2012; Cazzoli et al. 2014, 2016). The four free parameters of the model are the line 
centroid of the strongest blue absorption in the doublet $\left(\lambda_{1}\right)$, the line width $\left(\sigma_{1}\right)$, the amplitude of the blue component $\left(\mathrm{A}_{\lambda 5890}\right)$, and the ratio between the amplitudes of the blue and red components $\left(\mathrm{A}_{\lambda 5890} / \mathrm{A}_{\lambda 5896}=\alpha\right)$. The central blue wavelength of the $\mathrm{Na}$ I D doublet (Na I D 15890 ), $\lambda_{1}$ defines a single Doppler shift to respect the galaxy's systemic velocity (defined as the systemic redshift, $\lambda=5890 \AA$ ),$\Delta V_{1}$. The ratio between the two amplitudes is limited to vary between an optically thin absorbing gas, $\alpha=2$, and an optically thick absorbing gas, $\alpha=1$ (Spitzer 1978).

This approach allows us to characterize the kinematics of the global observed doublet without introducing other model dependent parameters. However, some stacked spectra show complex profiles, that suggest the presence of an additional Gaussian pair, in emission or in absorption (e.g. Chen et al. 2010; Cazzoli et al. 2016). This is motivated by two main factors. First, the scattering processes that arise in the flowing material can produce $P$ Cygni-like profiles of $\mathrm{Na} \mathrm{I} \mathrm{D} \mathrm{(see} \mathrm{the} \mathrm{analysis} \mathrm{of} \mathrm{the} \mathrm{absorption}$ and emission-line profiles for the Mg II $\lambda 2796,2803$ doublet and Fe II multiplet at $\lambda 2600 \AA$ in cold gas wind models shown in Prochaska et al. 2011), characterized by blue-shifted absorption plus resonance line emission at roughly the systemic velocity.

To model both the P Cygni profile and the interstellar absorption or emission at the systemic velocity, we introduce a second pair of Gaussians at the one - free - doublet model.

In particular, we define two new models of double doublets, the two - free - fix - doublet and the two - free - doublet, respectively model 2 and 3 .

Model 2. The two-free-fix-doublet consist of two Gaussian doublets; one is fixed to the systemic velocity and one is free to vary in the wavelength space. The model is described by the line centroid of the two doublets $\left(\lambda_{1}\right.$ and $\left.\lambda_{2}\right)$, the lines widths $\left(\sigma_{1}\right.$ and $\left.\sigma_{2}\right)$, the amplitudes of the blue lines $\left(\mathrm{A}_{\lambda 5890} 1\right.$ and $\left.\mathrm{A}_{\lambda 5890} 2\right)$, and the ratios $\alpha_{1}$ and $\alpha_{2}$. We note that often the two - free - doublet leads to unphysical solutions since the absorption or emission troughs are not well resolved. In order not to create this spurious result, the width of the second pair of Gaussians is constrained to the velocity dispersion of the nebular gas previously fitted to the $\mathrm{H} \alpha$ emission line (similar to those assumed in previous studies, e.g. Cazzoli et al. 2016 and Martin et al. 2012b, by using the [OII] doublet). The model described the observed line with five free parameters. As in the first model the flux ratios $\left(\alpha_{1}\right.$ and $\left.\alpha_{2}\right)$ are set to vary between 1 and 2 . The velocity of the outflowing gas is now expressed by the shift of the central blue wavelength $\left(\lambda_{1}\right), \Delta V_{2}$.

Model 3. The two - free - doublet is the same as model 2 , but with both the doublets free to vary in the wavelength range (seven free parameters). In this way, also the non-systemic red component (in emission or absorption) is well fitted. The blueshift of the outflowing component is referred to as $\Delta V_{3}$.

To avoid overfitting and to allow the correct number of Gaussian doublets used in each $\mathrm{Na}$ I D line fit, we employed the the Bayesian information criterion (BIC; Liddle 2007). BIC is a likelihood criterion penalized by the model complexity widely used in the problem of model identification. This method is particularly useful when a set of different candidate models with a different number of parameters is used to describe a given data set. The BIC criterion is defined as BIC $=\chi^{2}+p * \ln (n)$, where $\chi^{2}$ is the chi-square of the fit, $p$ is the number of free parameters, and $n$ is the number of flux points used in the fit. We measured the BIC value by using the three fitting methods described above, BIC1, BIC2, and BIC3, respectively. The model with the smallest value of the BIC was chosen as the preferred model for the data. We visually inspected each fit to evaluate the accuracy of this procedure. According to this statistical method we used $\Delta V_{1}$,
$\Delta V_{2}$, and $\Delta V_{3}$ and $\sigma_{1}, \sigma_{2}$, and $\sigma_{3}$ of the fit with the lowest BIC value to quantify the blueshift and the width of the outflowing component.

In order to estimate measurements uncertainties, the observed spectrum was perturbed randomly accordingly to the observed S/N. In this way we simulated 1000 different realizations of the same observed spectrum. We fitted the obtained noisy spectra with our three methods. Each quantity $\left(\Delta V_{1}, \Delta V_{2}\right.$ , $\Delta V_{3}, \sigma_{1}, \sigma_{2}$, and $\sigma_{3}$ ) was measured in all perturbed template spectra. The $68 \%$ confidence interval of each quantity distribution around the mean value was adopted as a measure of the uncertainty.

As in Rupke et al. (2005b), we defined a blue-shifted detection only if the difference between the velocity peak and the systemic velocity was $>70 \mathrm{kms}^{-1}$ (instrumental resolution of our SDSS data). To better parameterize the blue wings of the neutral $\mathrm{Na} I \mathrm{D}$ velocities, we also provided a measure of the maximum velocity, $V_{\max }=\Delta V-2 \sigma$ as defined for the absorption $\mathrm{Na}$ I D component in Rupke \& Veilleux (2015).

\section{Results}

\subsection{Na I D lines in the global sample}

After subtraction of the stellar continuum, we measured the residual equivalent width, $\mathrm{EW}(\mathrm{NaD})$, in all the 148 stacked spectra of the $\mathrm{C} 17$ Sample. Figure 4 shows the $\mathrm{EW}(\mathrm{NaD})$ obtained for each stacked spectrum in the SFR- $M_{\star}$ plane. As was found for individual galaxy spectra in Fig. 2, the mean EW(NaD) for the stacked spectra also increases progressively from negative values (emission) to positive values (absorption) as a function of the $M_{\star}$ and with no dependence on the SFR up to $\sim 4-5 \times 10^{10} M_{\odot}$. At the high-mass end $\left(>5 \times 10^{10} M_{\odot}\right)$, we observed a different $\mathrm{EW}(\mathrm{NaD})$ as a function of the SFR. The EW(NaD) reaches its maximum in the starburst region at high SFR and in the quiescent region at very low SFR with a mean value of $\mathrm{EW}(\mathrm{NaD}) \sim 0-0.25 \AA$ on the main sequence (MS) and in the transition region, respectively. By looking at the individual spectra it is possible to identify key differences in the Na I D line profile.

We applied the fitting procedure described in Sect. 3.2 to all 148 stacked spectra in order to derive the neutral gas kinematics expressed through the $\Delta V$ and $\sigma$ parameters. To facilitate the view, we isolated three examples of representative regions in the diagram: one at very low stellar masses (region A), one at high stellar mass and SFR (region B), and one at high stellar mass and low SFR (region C). The $\mathrm{Na} I \mathrm{D}$ region of the three spectra is shown in Fig. 5. In particular, the left panels show the observed spectrum (black curve) and the best fit stellar contribution (red curve), while the right panels show the ISM residual obtained after removal of continuum+emission lines. As previously reported by Jeong et al. (2013), we find that lowmass galaxies at stellar masses below $\sim 4-5 \times 10^{10} M_{\odot}$ and at any SFR are characterized by a residual $\mathrm{Na}$ I D emission at the galaxy systemic velocity (top panels of Fig. 5), likely arising from HII regions (e.g. Bica \& Alloin 1986). At higher stellar masses, $M_{\star}>5 \times 10^{10} M_{\odot}$ and high SFR, the EW(NaD) increases to positive values and shows an indication of a slightly blueshifted absorption (central panels of Fig. 5). At high $M_{\star}$ and low $\mathrm{SFR}$, in the quiescent region (region $\mathrm{C}$ ), we observed a residual Na I D absorption centred at the galaxy systemic velocity (bottom panels of Fig. 5). The existence of a $\mathrm{Na} I \mathrm{D}$ residual absorption in the early-type galaxies is known (e.g. Carter et al. 1986; Alloin \& Bica 1989; Thomas et al. 2003; Worthey 1998; Worthey et al. 2011) and has been largely debated. This could 


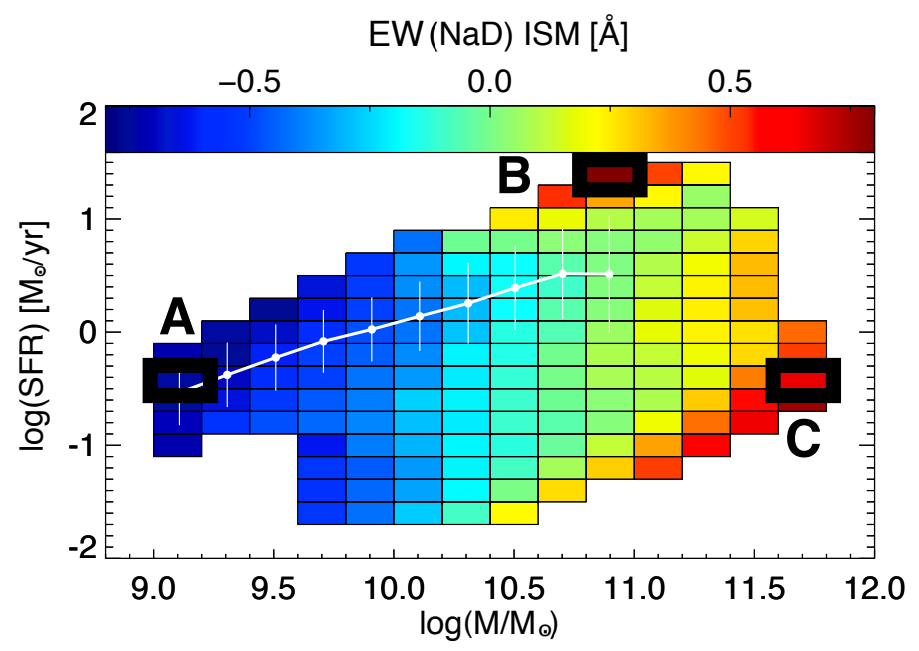

Fig. 4. SFR vs. $M_{\star}$ plane for our DR7 SDSS galaxies, colour-coded by the intensity of the EW of the ISM NA I D line after the stellar continuum subtraction. Negative and positive EW values correspond to a residual emission and absorption feature, respectively. The white line shows the mode and dispersion of the MS (see Fig. 1). For the highlighted bins, A, B, and C, the Na I D line fit is shown in Fig. 5.

be due to an extra $\mathrm{Na}$ abundance in the ISM, or in the stellar atmospheres (e.g. O'Connell 1976; Peterson 1976; Parikh et al. 2018), or to a bottom-heavy stellar initial mass function (IMF) van Dokkum \& Conroy 2010, 2012; Spiniello et al. 2012). We confirm here that in any of the stacked spectra of the quiescence region, the extra $\mathrm{Na} I \mathrm{D}$ absorption is observed at the systemic velocity and does not show any signature of ongoing outflow.

To investigate the dependence of the residual $\mathrm{Na}$ I D line profile on the SFR, we stacked all the galaxies with $M_{\star}>$ $5 \times 10^{10} M_{\odot}$ in bins accoring to SFR. The results are shown separately for the whole sample, the "pure" SF galaxies, and the AGN in Fig. 6. We find that statistically the Na I D residual absorption is observed above the $3 \sigma$ level and with a significant blueshift $\left(\Delta V>100 \mathrm{kms}^{-1}\right)$ only at SFR $\geq 10-12 M_{\odot} \mathrm{yr}^{-1}$, which is consistent with the starburst region above the local MS (Renzini \& Peng 2015). This is obtained independently of the nuclear activity, because the trend is the same for pure SF galaxies and AGN hosts separately (central and bottom panels of Fig. 6, respectively). We note that, to be conservative, the $\sigma$ level used to estimate the significance of the $\mathrm{Na} I \mathrm{D}$ residual absorption is estimated in a much larger region of the galaxy spectrum shown in Fig. 6. This is done to properly take into account the uncertainty of the stellar continuum subtraction.

We also note that the level of significance of the Na I D absorption at level of SFR in the range $1-10 M_{\odot} \mathrm{yr}^{-1}$ could be biased by the contribution of a non-negligible percentage of systems with Na I D in emission. At $M_{\star}$ above $\sim 5 \times 10^{10} M_{\odot}$ and SFR 1-10 $M_{\odot} \mathrm{yr}^{-1}$ this percentage could be of the order of 30-40\%, as shown in Fig. 2. Thus, emission and absorption contributions could cancel each other out in the stacking, thus, leading to the low significance absorption at SFR in the range 1-10 $M_{\odot} \mathrm{yr}^{-1}$ (upper panel of Fig. 6).

\subsection{Cold winds in starburst galaxies}

Based on the results shown in the previous section, we focus on galaxies located at SFR $\geq 12.5 M_{\odot} \mathrm{yr}^{-1}$ where there is a clear signature of blue-shifted interstellar Na I D absorption. We refer to this sub-sample as the starburst sample; it contains 15284
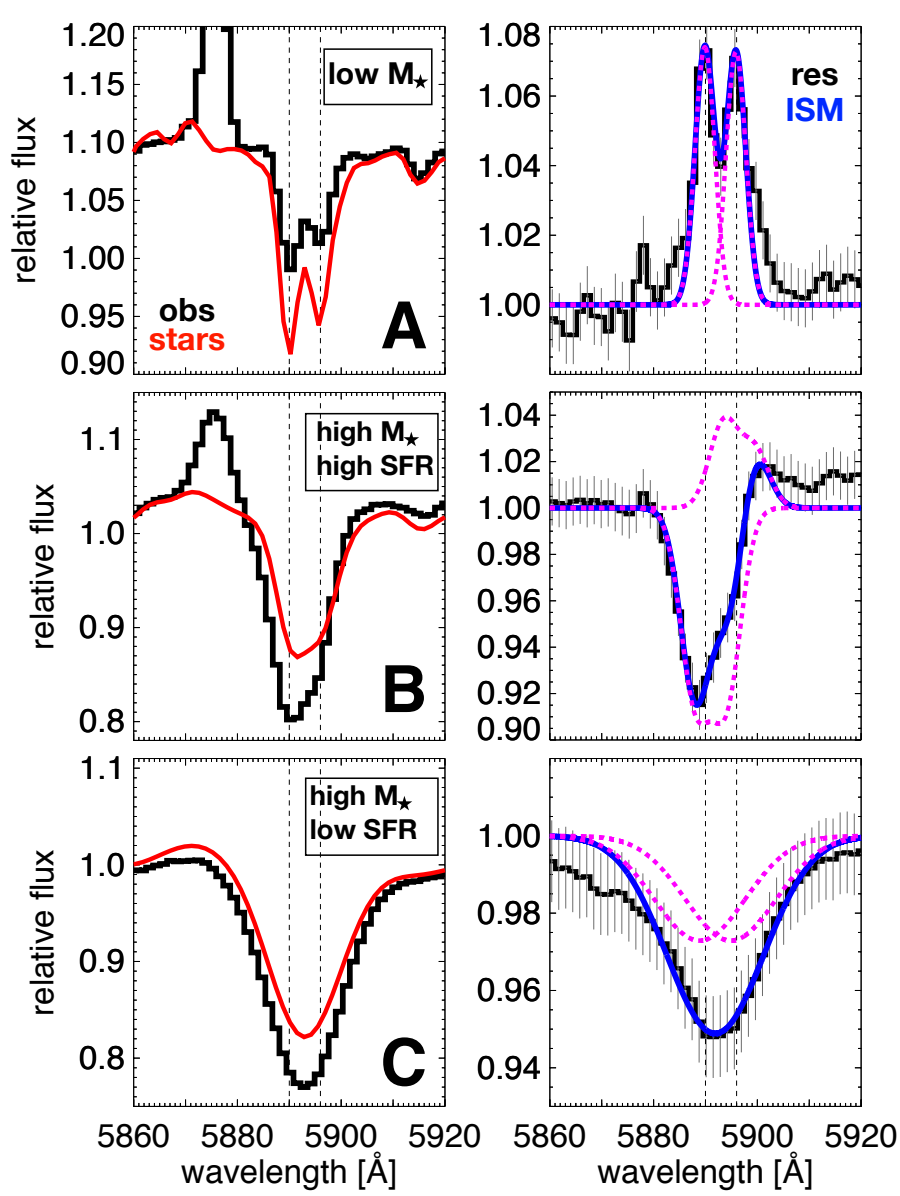

Fig. 5. Examples of Na I D fits of the stacked spectra from three different regions of the SFR- $M_{\star}$ plane, labelled in Fig. 4: low stellar mass $\left(\log (M)_{\star}<10.5 M_{\odot}\right.$, top panels, bin A in Fig. 5), high stellar mass and high SFR $\left(\log (M)_{\star}>10.5 M_{\odot} \log (\mathrm{SFR})>1.1 M_{\odot} \mathrm{yr}^{-1}\right.$, central panels, bin B in Fig. 5), and low SFR and high stellar mass $\left(\log (M)_{\star}>10.5 M_{\odot}, \log (\mathrm{SFR})<0.5 M_{\odot} \mathrm{yr}^{-1}\right.$, bottom panels, Bin $\mathrm{C}$ in Fig. 5). The expected wavelengths of $\mathrm{Na} \mathrm{I} \mathrm{D} \mathrm{are} \mathrm{shown}$ as vertical dashed lines. Left panels: black histogram illustrates the observed stacked spectrum, the red line the best-fit stellar continuum. Right panels: black histogram shows the residual flux and errors after the stellar continuum has been removed. The best-fit of the doublet is shown in blue. The dotted magenta curves are the single-Gaussian components. Only in bin B (central panel) do we detect a Doppler blueshift of the $\mathrm{Na}$ I D absorption feature with $\Delta V=-102 \pm 41 \mathrm{~km} \mathrm{~s}^{-1}$. In the other two bins the Na I D line shows a zero-velocity emission (bin A, top panel) and absorption (bin C, bottom panel) below the instrumental resolution $70 \mathrm{~km} \mathrm{~s}^{-1}$.

galaxies. We investigate the relation between the significance of the $\mathrm{Na}$ I D absorption and its blueshift with respect to (i) galactic disc inclination to identify the wind geometry, (ii) the galaxy nuclear activity to identify the ejection mechanism, and (iii) the concurrence with the ionized wind to better study the multiphase nature of the wind. In the following analysis we apply the fitting procedure described in Sect. 3.2.

In order to study the wind geometry, we split the starburst sample into six bins according to inclination angle taken from the bulge/disc decomposition of the Simard et al. (2011) catalogue (see Table 1 for the subsample information). Figure 7 illustrates the interstellar $\mathrm{Na}$ I D absorption profiles for composite spectra in six different inclination bins. We find a clear trend between the blue-shifted absorption profiles and the galaxy inclination: the neutral sodium line shows a transition from a strong 

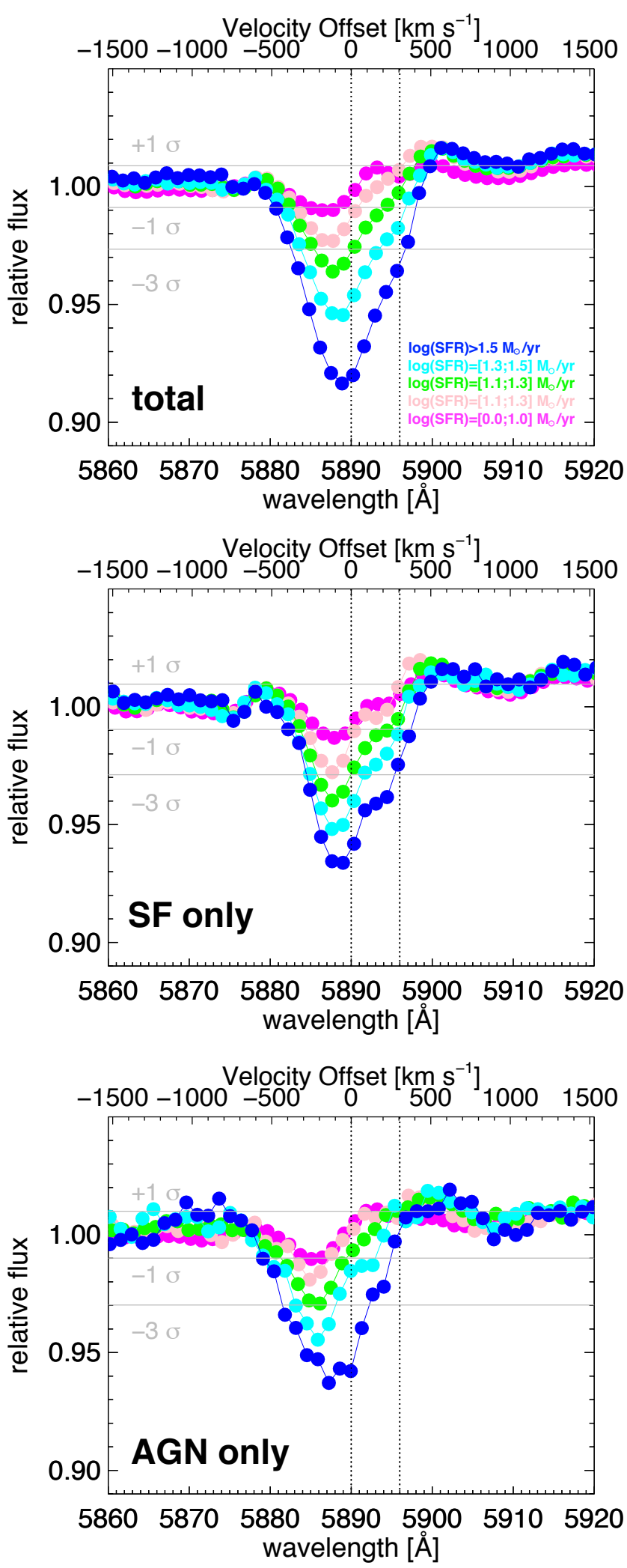

Fig. 6. Variation in the ISM Na I D resonant line profile as a function of the SFR for the galaxies with $\log (M)_{\star}>10.7 M_{\odot}$ and $\log (\mathrm{SFR})>$ $0.0 M_{\odot} \mathrm{yr}^{-1}$ in the total sample (top panel), in pure SF galaxies (middle panel) and in AGN dominated objects (bottom panel). The intensity of the line increases with increasing SFR, from the magenta to the blue curves. Only galaxies with SFR $\geq 12 M_{\odot} \mathrm{yr}^{-1}$ show a blue-shifted $\mathrm{Na}$ I D line, detected with a significance $>3 \sigma$. The pure $\mathrm{SF}$ and AGN subsamples show the same trend observed in the total sample.
Table 1. Basic data about the starburst sample (galaxies with SFR > $10^{1.1} M_{\odot} \mathrm{yr}^{-1}$ ) slitted in six bins of inclination angle taken from Simard et al. (2011).

\begin{tabular}{lcc}
\hline \hline Subsample & Number & Percent \\
\hline TOT & 8772 & $100 \%$ \\
\hline$i=[0-30]$ & 1684 & $19.2 \%$ \\
$i=] 30-40]$ & 1438 & $16.4 \%$ \\
$i=] 40-50]$ & 1769 & $20.2 \%$ \\
$i=] 50-60]$ & 1779 & $20.3 \%$ \\
$i=] 60-70[$ & 1336 & $15.2 \%$ \\
$i=[70-90]$ & 766 & $8.7 \%$ \\
\hline
\end{tabular}

disc-like component, perfectly centred to the systemic velocity in the edge-on system (inclination $i>50^{\circ}$ of the disc rotation axis) to an outflow, blue-shifted component in face-on galaxies $\left(i<50^{\circ}\right)$. This result is perfectly consistent with the finding of Chen et al. (2010) for a subsample of SF galaxies. We note that in our edge-on systems the stellar continuum light is strong enough to guarantee a robust background continuum illumination, which is essential in order to measure the ISM absorption.

Figure 8 shows the mean velocity shift (top panel), velocity dispersion (central panel), and relative efficiency of the parameterized models expressed by the BIC value (bottom panel) as a function of the disc orientation. We find that the $\mathrm{Na}$ I D lines in highly inclined galaxies can be reproduced with the one - free - doublet model (filled circles in the figure). The velocity of the line peak is consistent with the systemic velocity of the galaxy $\left(\Delta V \sim 0 \mathrm{kms}^{-1}\right)$. In the low-inclination spectra, we find a very complicated $\mathrm{Na}$ I D line structure. In particular, we find evidence of the presence of a blue-shifted absorption component and a line-emission at roughly the systemic velocity; this is consistent with the P Cygni predictions shown in the cool gas wind models for the $\mathrm{Mg}$ II and Fe II resonance lines of Prochaska et al. (2011; Monte Carlo radiative transfer techniques) and Scarlata \& Panagia (2015; semi-analytical line transfer model). For these low-inclination galaxies methods 2 and 3 better reproduce the line shape, as is shown in the bottom panel of Fig. 8 by the low BIC values. In the face-on galaxy sample, the velocity of the line peak reaches a maximum value of $\Delta V \sim 200 \mathrm{kms}^{-1}$ and maximum velocity of $V_{\max } \sim 460 \mathrm{kms}^{-1}$. Instead, the line width does not show, any dependence on the disc inclination and has a constant value of $\sigma \sim 130 \pm 30 \mathrm{kms}^{-1}$ (see central panel of Fig. 8). This could likely be due to the stacking procedure, which averages out all velocity structure information.

We find that the trend with galaxy inclination at fixed SFR is present irrespective of the contribution of nuclear activity. In all the ionization classes (SF, SFAGN, AGNSF, and AGN) we observe the same trend as shown in Fig. 7 at SFR $>10 M_{\odot} \mathrm{yr}^{-1}$. The right panels of Fig. 9 show, in particular, the Na I D line profile for pure SF galaxies (upper panel) and AGN hosts (lower panel) in two bins of inclination $\left(i<50^{\circ}\right.$, green curve, and $i>50^{\circ}$, magenta curve). Highly inclined systems show no velocity shift, while face-on SF galaxies and AGN hosts exhibit on average the same velocity blueshift $\Delta V \sim 200 \mathrm{~km} \mathrm{~s}^{-1}$.

\subsection{Neutral versus ionized wind}

To analyse the concurrence of neutral gas and ionized gas winds, we compare here the $\mathrm{Na}$ I D absorption line shape with the emission line profile of the [OIII] $\lambda 5007, \mathrm{H} \alpha,[\mathrm{NII}] \lambda 6548$, and $[\mathrm{NII}] \lambda 6584$, whose perturbed kinematics is generally used as tracer of ionized gas outflows (see e.g. Concas et al. 2017; 


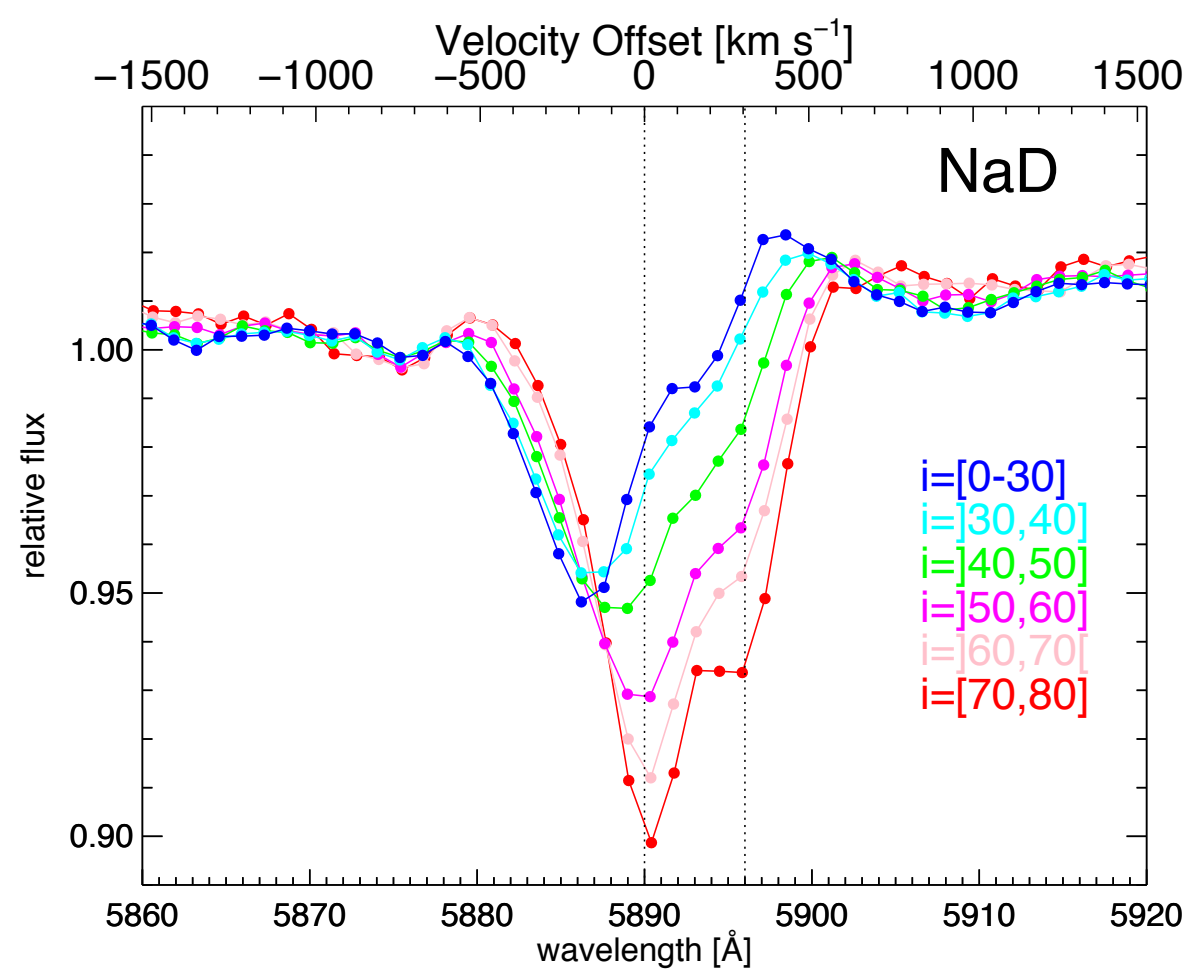

Fig. 7. Variation in the ISM Na I D resonant line profile as a function of galaxy inclination, for the galaxies with SFR $\geq 10^{1.1} M_{\odot} \mathrm{yr}^{-1}$ (including pure SF galaxies and galaxies with an AGN contribution). The line is centred to the systemic velocity in the edge-on sample and shows a progressive blueshift as the mean disc inclination decreases. The maximum shift is reached by face-on galaxies (blue curve).
Perna et al. 2017a; Mullaney et al. 2013; Harrison et al. 2014). This comparison is done only for the starburst galaxy sample, where we observe a clear signature of neutral gas outflow. The analysis is performed as a function of the ionization class and disc inclination.

The presence of outflow in the emission line profile is identified through the contribution of a second blue-shifted emission component in addition to a narrow component at the systemic velocity. In this respect, we apply here the same procedure described in $\mathrm{C} 17$. The [OIII] $\lambda 5007$ line profile, in particular, is fitted either with a single Gaussian or with a combination of two Gaussians. The best fit is chosen according to the BIC, as explained in Sect. 3.2. The decomposition of the $\mathrm{H} \alpha$, [NII] 66548 , and [NII] $\lambda 6584$ emission lines is made more complicated by the proximity of all features. The blending of these lines makes it impossible to carefully constrain the kinematics of the second broad and blue-shifted component. However, by using the results obtained for the [OIII] line fit (line width and shifts of the different components) and leaving the line amplitude free to vary, we are able to successfully reproduce the $\mathrm{H} \alpha$ region (see the line fit in right panel of Fig. 9). We note that the second broad component in the $\mathrm{H} \alpha$ emission line could also be associated with photon emission originating from the broad line regions of AGN galaxies. The proximity of nitrogen emission lines and this possible contamination means that the study of the ionized outflow signatures in our SDSS spectra cannot be performed by using the $\mathrm{H} \alpha$ emission line region alone.

Similarly to what was found in C17, we do not observe signature of ionized gas wind in pure SF galaxies. This is true irrespective of the disc inclination. The upper panels of Fig. 9 show the stacked [OIII] $\lambda 5007$ (central panel) and $\mathrm{H} \alpha$, [NII] $\lambda 6548$, and [NII] $\lambda 6584$ (right panel) profiles in two bins of inclination ( $i<50^{\circ}$, green curve, and $i>50^{\circ}$, magenta curve). While faceon SF galaxies exhibit a clear sign of neutral gas wind, no outflow is ongoing in the ionized gas phase.

In addition, we also confirm that the significance of outflow signature of ionized gas increases at increasing AGN con- tribution, with the highest significance for the AGN class. The overall line width of the [OIII] line increases from a FWHM of $\sim 300 \mathrm{kms}^{-1}$ (corresponding to $\sigma \sim 127 \mathrm{kms}^{-1}$ ) for pure SF galaxies to $\sim 600 \mathrm{kms}^{-1}\left(\sigma \sim 257 \mathrm{kms}^{-1}\right)$ for AGN hosts, at fixed $M_{\star}$ and SFR. The same is found for the $\mathrm{H} \alpha$, [NII] $] 6548$, and [NII] 26584 line profiles. The bottom centre and right panels of Fig. 9 clearly indicate the presence of a second broad and blueshifted Gaussian component in all emission lines of the AGN hosts. However, while the neutral gas wind is observed only in face-on active galaxies, the ionized gas outflow does not show any dependence on the disc inclination in this class of objects.

The evidence for multiphase gas wind only in the face-on AGN population is in agreement with the results obtained by Rupke et al. (2005b), who find agreement between neutral and ionized wind kinematics in Seyferts but not in starburst systems. Similarly, Rupke et al. (2017) find a high coincidence rate $(50 \%$ of the sample) of ionized and neutral gas winds in a sample of ten nearby Type 1 quasars. Differently, Villar-Martín et al. (2011) do not find any clear evidence of neutral outflows in a subsample of 21 nearby SDSS QSO2. We note that our outcome is not directly in disagreement with the Villar-Martín et al. (2011) findings. The tension between the two results could be due to the small size of the Villar-Martín et al. (2011) sample and/or to the high galactic disc inclination of the 21 QSO.

\section{Discussion}

\subsection{Triggering mechanism: AGN or SF activity?}

As mentioned in the Introduction, in the current paradigm of galaxy formation, two main physical processes are advocated to sweep away the galaxy gas supply: (1) the AGN and (2) the SF activity itself. In the "ejective feedback" scenario, both mechanisms are supposed to be able to push the gas away via powerful winds, reduce the gas reservoir, and therefore stop the growth of the galaxies. From an empirical point of view, the most important issue in our understanding of galactic winds 


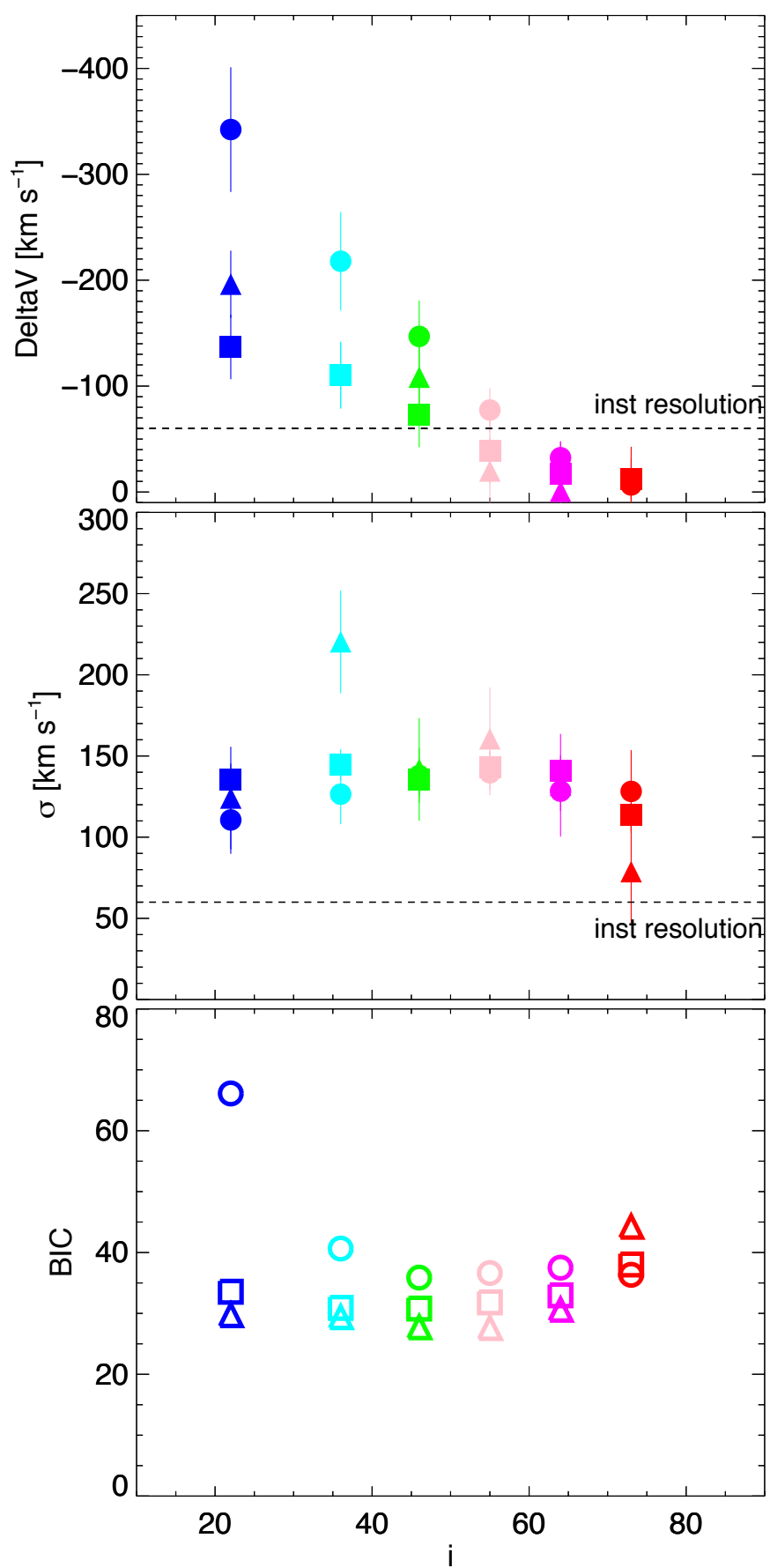

Fig. 8. Na I D velocity shift, velocity dispersion, and BIC value estimates determined via the three methods (circles, squares, and triangles for method 1, 2, and 3 respectively) described in Sect. 3 as a function of the inclination angle, from face-on to edge-on systems (blue and red, respectively). The peak velocity increases towards lower inclination angles. The width of the outflowing component is reasonably stable for all the methods adopted with a mean value around $\sigma \sim 130 \pm 30 \mathrm{kms}^{-1}$ The BIC associated with a single doublet component, method 1 , is favoured only at large inclination values, while methods 2 and 3 provide a better fit in the other cases.

is the relative importance of SF- and AGN-driven winds (e.g. Rupke \& Veilleux 2013a).

As shown in previous sections, we find that the neutral gas winds as traced by Na I D absorption lines are connected with the SFR without any correlation with the presence of an optically selected AGN. The neutral gas wind could be originated only in galaxies with very high SF activity. In addition, as we showed in Sect. (Trends with galaxy inclination), the clear inclination dependence of the neutral winds strongly suggests that the outflowing material is mainly ejected from the galactic disc where the SF regions are located.

Instead, by observing the ionized gas phase in the same galaxy spectra, we find that ionized outflows, as traced by [OIII], $\mathrm{H} \alpha$, [NII] $\lambda 6548$, and [NII] $\lambda 6584$ emission lines, are present on average only in the AGN sample, while they are absent in the SF sample (centre and right panels of Fig. 9). This difference was first pointed out in Concas et al. (2017) for the [OIII] emission line, and it is strengthened in this work by the analysis of the $\mathrm{H} \alpha$ and $[\mathrm{NII}]$ emission lines complexes. That ionized outflows appear only in AGN systems and that they are independent from the galactic disc inclination strongly supports the AGN origin of these winds.

We can conclude that in the local Universe, different gas phases of the ISM undergo wind events of different natures: the neutral $\mathrm{Na} I \mathrm{D}$ outflowing gas is mainly ejected from the galactic disc above a threshold of SF activity, while the ionized gas outflow traced by the [OIII], $\mathrm{H} \alpha$, [NII] $\lambda 6548$, and [NII] $\lambda 6584$ line asymmetry is likely powered by AGN feedback.

As suggested in several papers (e.g. Veilleux et al. 2005; Rupke \& Veilleux 2005, 2013b; Cicone et al. 2014; Fluetsch et al. 2018), the energetics of the outflowing gas are more extreme in galaxies that host a powerful AGN compared to the normal starburst systems. For this reason, a common way to discriminate between the SF- and AGN-driven winds is to compare the kinetic and momentum power $\left(\dot{E}_{w}\right.$ and $\dot{P}_{w}$, respectively) associated with the winds with the AGN bolometric luminosities and the kinetic power expected to be ascribed to stellar processes (e.g. Veilleux et al. 2005; Brusa et al. 2015).

The kinetic and momentum powers of the galactic winds can be expressed as (see Cano-Díaz et al. 2012; Cresci et al. 2015)

$\begin{aligned} \dot{E}_{w} & =\frac{1}{2} \dot{M}_{\text {out }} v_{\text {out }}^{2}, \\ \dot{P}_{w} & =\dot{M}_{\text {out }} v_{\text {out }},\end{aligned}$

where $v_{\text {out }}$ is the velocity of the ejected gas and $\dot{M}_{\text {out }}$ is the mass outflow rate. Following Cano-Díaz et al. (2012), the mass outflow rate can be defined as

$\dot{M}_{\text {out }}^{[\mathrm{OIII}]}=0.48 \times \frac{C L_{[\mathrm{OIII}]} v_{\mathrm{out}}}{n_{\mathrm{e}} R_{\mathrm{kpc}} 10^{[\mathrm{O} / \mathrm{H}]}} M_{\odot} \mathrm{yr}^{-1}$,

where $C$ is the condensation factor $(C \sim 1), L_{[\mathrm{OIII}]}$ is the oxygen luminosity associated with the wind component in units of $10^{41} \mathrm{erg} \mathrm{s}^{-1}, 10^{[\mathrm{O} / \mathrm{H}]}$ is the gas metallicity in solar units, $n_{\mathrm{e}}$ is the electron density, and $R_{\mathrm{kpc}}$ is the radius of the extended wind in units of kpc. In the neutral gas counterpart, the mass flow rate can be estimated as

$\dot{M}_{\text {out }}^{\mathrm{H}}=N_{\mathrm{H}} \times R_{5 \mathrm{kpc}} \times v_{\text {out } \_300} M_{\odot} \mathrm{yr}^{-1}$,

where $N_{\mathrm{H}}$ is the hydrogen column density in units of $10^{20} \mathrm{~cm}^{-2}$, $R_{5 \mathrm{kpc}}$ is the radius of the extended wind in units of $5 \mathrm{kpc}$, and $v_{\text {out } 300}$ is the velocity of the ejected gas in units of $300 \mathrm{kms}^{-1}$ (see Weiner et al. 2009; Perna et al. 2015).

In the ionized and in the neutral gas phases, the estimate of the mass outflow rate depends on several assumptions, in particular on the geometry of the winds (biconical, spherical, or more complex), their extent $(R)$, and the density of the material embedded on the gas flow $\left(n_{\mathrm{e}}\right.$ and $\left.N_{\mathrm{H}}\right)$.

For electron density recent literature results converge on a typical value of $n_{\mathrm{e}} \sim 1000 \mathrm{~cm}^{-3}$ (see Perna et al. 2017a; 
$\mathrm{NaD}$

[OIII]

$\mathrm{H} \alpha+[\mathrm{NII}]$
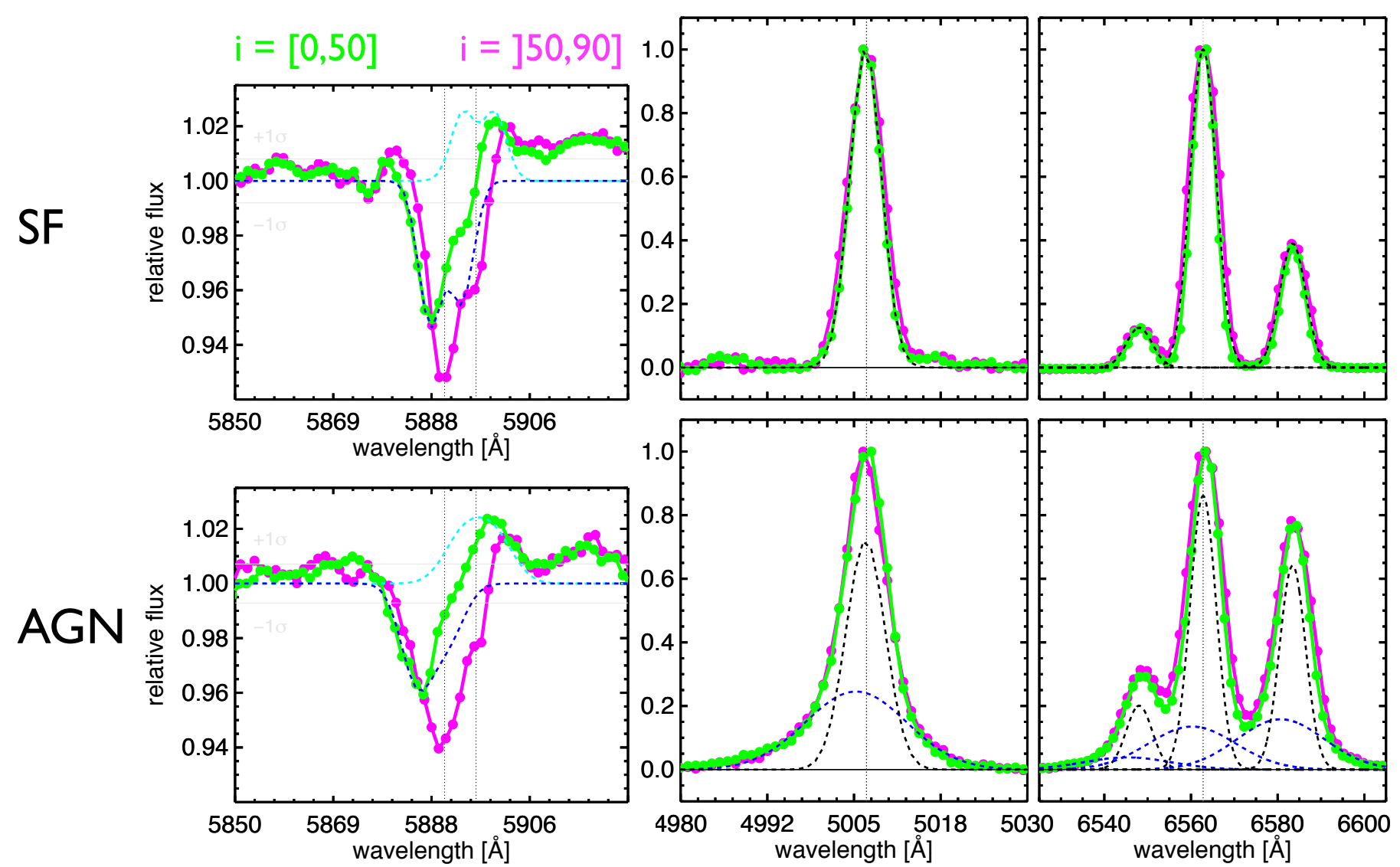

Fig. 9. Correlation between the $\mathrm{Na}$ I D resonant line, [OIII $] \lambda 5007$ and $\mathrm{H} \alpha$ emission-line profiles (left, central, and right panels, respectively) and the galaxy stellar disc inclination (face-on and edge-on systems with inclination $i=[0,50]$ and $i=] 50,90]$ in green and magenta, respectively). The spectra are extracted from the pure SF galaxies (top panels) and for the AGN subsample (bottom panels). The black and blue dashed lines show the decomposition between the narrow systemic and the broad blue-shifted components needed to best fit the data. In the sodium panels, the cyan dashed lines illustrate the emission line component consistently with the P Cygni predictions shown in the cool gas wind models of Prochaska et al. (2011) and Scarlata \& Panagia (2015). The Na I D line shift clearly correlates with the galaxy inclination in both SF and AGN samples. The [OIII] and $\mathrm{H} \alpha$ emission lines are independent from the galaxy morphology, but show a strong shape variation between the SF and AGN subsamples.

Table 2. Basic data about the starburst sample (galaxies with SFR > $12.5 M_{\odot} \mathrm{yr}^{-1}$ ) slitted in five bins of ionization mechanisms.

\begin{tabular}{lcc}
\hline \hline Subsample & Number & Percent \\
\hline TOT & 15284 & $100 \%$ \\
\hline SF & 3735 & $24.4 \%$ \\
SF-AGN & 4196 & $27.4 \%$ \\
AGN-SF & 3912 & $25.6 \%$ \\
AGN & 872 & $5.7 \%$ \\
unClass & 2569 & $16.8 \%$ \\
\hline
\end{tabular}

Förster Schreiber et al. 2018); instead, the hydrogen column density in the neutral gas phase are more difficult to estimate, especially in stacked spectra. By stacking hundreds different galaxy spectra, we have averaged together galaxies with a wide range of optical depth and cover fraction distributions that are essential for a correct estimate of $N_{\mathrm{H}}$. In addition, in both the neutral and ionized phases it is impossible to determine the exact extension of our outflowing gas. Our spectra are extracted within a $3^{\prime \prime}$ diameter region, so we can provide at most an upper limit to the wind extension, and thus only a lower or upper limit to the ionized and neutral mass outflow rates, respectively. Moreover, given the relatively large redshift range of our sample, the physical scale sampled varies by $\sim 2$ orders of magnitude within the single sources, which makes up the stacked spectrum and makes it impossible to make even an order of magnitude estimate of the neutral and ionized powers inside the winds. To measure the dimension and also the geometry of such winds is mandatory in order to spatially resolve the outflow (see the recent integral field spectroscopy analysis, IFS, in Rupke et al. 2017 and Venturi et al. 2018).

Nevertheless, the studies based on stacked spectra are essential to infer how common these outflowing phenomena are and how they are related to the galaxies properties. For all these reasons, they represent the crucial step to plan dedicated follow-up for new IFS investigations (e.g. the combined study of ionized wind in local optically selected AGN presented in Mullaney et al. 2013 and Harrison et al. 2014).

\subsection{Effect on baryon cycle}

As shown in Sect. 4, in both the neutral and the ionized phase, the outflow velocity is relatively small, of the order of 200 and $500 \mathrm{kms}^{-1}$ for the peak velocity shift and the maximum velocity, respectively. We use the catalogue of halo masses of 
Yang et al. (2007) to retrieve the mean halo mass of the pure SF and AGN hosts. The region of the SFR- $M_{\star}$ diagram considered here, at high stellar masses and SFR, is dominated at $60-70 \%$ by central galaxies of massive halos of $10^{12.5-13} M_{\odot}$, whose escape velocity $\left(v_{\text {escape }} \sim 700-1000 \mathrm{~km} \mathrm{~s}^{-1}\right)$ largely exceeds the outflow velocity observed here. Thus, we conclude that on average the outflowing gas will never escape the halo potential well. Cazzoli et al. (2016) found very consistent results in the analysis of $\sim 50$ local LIRGs and ULIRGs.

These low outflow velocities suggest that the neutral and ionized phases of the outflowing gas remain bound to the galaxy and likely fall back to the disc. These "light breezes" are consistent with the "gas circulation" scenario proposed by Fraternali \& Binney (2006; see also Fraternali 2017 for a review) for the Milky Way. In this scenario, the cold gas driven out of the disc by SF feedback mixes with the hot halo gas. The gas mixture cools down through metal lines emission and falls back to the disc with a ballistic trajectory, creating what is known as the galaxy fountain. In this picture the gas outflow does not stop the galaxy star formation activity; on the contrary, it sustains the process by triggering the cooling of the hot coronal gas and so further accretion.

We do not find, however, any evidence of accreting recycling gas in any of the analysed stacked spectra. This may be related to the predicted low velocities of the inflowing material. For typical spiral galaxies, the fountain gas is expected to fall back at velocities of the order of $70-100 \mathrm{kms}^{-1}$ or less (as predicted by Fraternali \& Binney 2006 for NGC 891 and by Marasco et al. 2012 for the Milky Way), which are comparable to the velocity resolution of the SDSS spectra. Furthermore, as discussed by Fraternali (2017), the fountain gas could fall back at larger radii with respect to the location where the peak of the SFR density occurs. The $3^{\prime \prime}$ diameter fibre in the SDSS spectra does not allow us to sample the more external region of the galaxies. Finally, recent parsec-scale hydrodynamical simulations, including the presence of thermal conduction, show that the efficiency of the fountain-driven gas accretion strongly decreases with increasing virial temperature of the halo gas (Armillotta et al. 2016). This effect can be particularly important for massive galaxies that reside in high-mass dark matter halos.

\section{Summary and conclusions}

In this paper, we have explored the presence of galactic neutral gas winds in a statistically significant sample of local galaxies drawn from the SDSS, including normal and active galaxies, in passive, star-forming, and starburst hosts. We used, in particular, the Na I D absorption feature as a tracer of cold neutral gas outflow. We interpreted the detection of a blue-shifted component with respect to the galaxy systemic velocity as evidence of outflowing gas, and we measured its mean and maximum outflow velocity. The single SDSS spectra are stacked together in bins of (a) SFR and $M_{\star}$ to study the neutral gas wind occurrence as a function of the galaxy position with respect to the MS of SF galaxies, (b) main photoionization mechanism (AGN or SF activity, or a combination of the two) to identify the most likely ejecting mechanism (SF or AGN feedback), and (c) galaxy inclination to investigate the outflow geometry. In each stacked spectrum the stellar continuum is carefully subtracted in order to remove the Na I D stellar absorption component and so enhance the residual ISM component. The main results of the paper can be summarized as follows:

- The neutral gas winds traced by the Na I D line are not ubiquitous in the SFR- $M_{\star}$ plane. A clear signature is detected in massive $\left(M_{\star}>5 \times 10^{10} M_{\odot}\right)$ highly SF galaxies (SFR > 10-12 $\left.M_{\odot} \mathrm{yr}^{-1}\right)$. However, we do not exclude the presence of an outflow signature in less SF galaxies at fixed mass, where the evidence could be hidden by the stacking of a similar percentage of $\mathrm{Na}$ I D in emission and absorption. In all other loci of the SFR- $M_{\star}$ plane the Na I D is observed either in emission (low stellar masses) or in absorption, but always at the systemic velocity (high stellar masses and low SFR), without exhibiting any evidence of outflow.

- The same results are obtained irrespective of the BPT classification: the AGN population follows the same trend in the SFR- $M_{\star}$ plane as the SF galaxies (see Fig. 6).

- We perform an in-depth analysis of the galaxies in the starburst region ( $\mathrm{SFR}>10 M_{\odot} \mathrm{yr}^{-1}$ ) at very high mass to study the geometry of the outflow. The $\mathrm{Na} I \mathrm{D}$ residual absorption shows a progressive blueshift with respect to the systemic velocity with the decrease in the disc inclination. The highest values of peak and maximum velocity shift ( $\Delta V \sim 200$ and $V_{\max } \sim 460 \mathrm{kms}^{-1}$, respectively) are observed in the lowinclination face-on galaxies, while no outflow is observed in edge-on systems. This would suggest that the outflow direction is perpendicular to the disc or that it exhibits a large opening angle.

- We find no strong correlation between the evidence of neutral gas wind and the nuclear activity. The $\mathrm{Na} I \mathrm{D}$ line profile of pure SF, SFAGN, AGNSF, and AGN samples shows the same velocity shift and the same dependence on disc inclination.

- In the face-on galaxies the $\mathrm{Na} I \mathrm{D}$ residual component profile is also characterized by a typical P Cygni profile as predicted by several outflow models (see Prochaska et al. 2011 and Scarlata \& Panagia 2015). This particular feature is also consistent with recent IFU observations of $\mathrm{Na}$ I D resonant lines in low-redshift QSO systems (e.g. Rupke \& Veilleux 2015 and Rupke et al. 2017).

- Pure SF galaxies do not show any evidence of multiphase gas wind. The observed cold gas outflows traced by the $\mathrm{Na}$ I D in galaxies at high stellar mass and SFR is not accompanied by any evidence of ionized gas phase wind traced by $[\mathrm{OIII}] \lambda 5007, \mathrm{H} \alpha,[\mathrm{NII}] \lambda 6548$, and [NII] $\lambda 6584$ emission lines.

- We find clear evidence of multiphase gas outflow only in the AGN population. However, while the Na I D blueshift strongly depends on the inclination and is greatest for face-on galaxies, the [OIII] $\lambda 5007, \mathrm{H} \alpha,[\mathrm{NII}] \lambda 6548$, and [NII] $\lambda 6584$ line profiles do not show any dependence on the galactic disc inclination. This suggest that the cold gas outflow is driven by SF feedback above a given SFR threshold, while the ionized gas outflow originates from the nuclear region. Its direction is likely related to the black hole accretion disc inclination which is decoupled from the host disc.

We note that our results do not exclude that strong neutral gas wind might be caused by $\mathrm{BH}$ feedback in powerful QSOs, as observed by Rupke et al. (2005a) and Rupke \& Veilleux (2011, 2013a, 2015), among others. However, such systems are very rare objects in the Local Universe and they do not dominate the mean in the stacking analysis presented in this work.

Finally, the low outflow velocities observed in both the neutral and ionized phases suggest that the outflowing gas remains bound to the galaxy and likely falls back to the disc with no definitive effect on the gas reservoir and on the galaxy SF activity. These light breezes observed in the SDSS galaxy spectra are 
consistent with the gas circulation scenario (Fraternali \& Binney 2006 and see Fraternali 2017 for a recent review). We do not find, however, any evidence of accreting re-cycling gas expected from a fountain cycle.

Much higher spectral and spatial resolution IFS observations are necessary to better understand the geometry of the moderate outflowing gas observed here, and to constrain its energetics, its nature, and its effect on the galaxy baryon cycle.

Acknowledgements. We thank the anonymous referee for the constructive and helpful report. AC thanks Federico Lelli for precious suggestions and stimulating discussion. AC, PP, and MB are grateful to the Munich Institute for Astrophysics and Particle Physics for its hospitality, where most of the discussions for this paper were carried out. This research was supported by the DFG cluster of excellence "Origin and Structure of the Universe".

\section{References}

Abazajian, K. N., et al. 2009, ApJS, 182, 543

Alloin, D., \& Bica, E. 1989, A\&A, 217, 57

Armillotta, L., Fraternali, F., \& Marinacci, F. 2016, MNRAS, 462, 4157

Baldry, I., Glazebrook, K., \& Driver, S. 2008, MNRAS, 388, 945

Baldwin, J. A., Phillips, M. M., \& Terlevich, R. 1981, PASP, 93, 5

Behroozi, P. S., Conroy, C., \& Wechsler, R. H. 2010, ApJ, 717, 379

Behroozi, P. S., Wechsler, R. H., \& Conroy, C. 2013, ApJ, 770, 57

Bica, E., \& Alloin, D. 1986, A\&A, 166, 83

Bordoloi, R., Lilly, S. J., Hardmeier, E., et al. 2014, ApJ, 794, 130

Bower, R. G., Benson, A., Malbon, R., et al. 2006, MNRAS, 370, 645

Brinchmann, J., Charlot, S., White, S., et al. 2004, MNRAS, 351, 1151

Brusa, M., Bongiorno, A., Cresci, G., Perna, M., et al. 2015, MNRAS, 446, 2394

Brusa, M., Perna, M., Cresci, G., et al. 2016, A\&A, 588, A58

Bruzual, G., \& Charlot, S. 2003, MNRAS, 344, 1000

Cano-Díaz, M., Maiolino, R., Marconi, A., et al. 2012, A\&A, 537, L8

Cappellari, M., \& Emsellem, E. 2004, PASP, 116, 138

Carter, D., Visvanathan, N., \& Pickles, A. J. 1986, ApJ, 311, 637

Cazzoli, S., Arribas, S., Colina, L., et al. 2014, A\&A, 569, A14

Cazzoli, S., Arribas, S., Maiolino, R., \& Colina, L. 2016, A\&A, 590, A125

Chabrier, G. 2003, PASP, 115, 763

Chen, Y.-M., Tremonti, C., Heckman, T., et al. 2010, AJ, 140, 445

Chevalier, R. A. 1977, ARA\&A, 15, 175

Cicone, C., Maiolino, R., Sturm, E., et al. 2014, A\&A, 562, A21

Cicone, C., Maiolino, R., \& Marconi, A. 2016, A\&A, 588, A41

Cicone, C., Brusa, M., Ramos Almeida, C., et al. 2018, Nat. Astron., 2, 176

Concas, A., Popesso, P., Brusa, M., et al. 2017, A\&A, 606, A36

Conroy, C., \& Wechsler, R. H. 2009, ApJ, 696, 620

Cresci, G., \& Maiolino, R. 2018, Nat. Astron., 2, 179

Cresci, G., Mainieri, V., Brusa, M., et al. 2015, ApJ, 799, 82

Croton, D. J. 2006, MNRAS, 365, 11

Davis, T. A., Krajnović, D., McDermid, R. M., et al. 2012, MNRAS, 426, 1574

De Lucia, G., Springel, V., White, S., Croton, D., \& Kauffmann, G. 2006 MNRAS, 366, 499

Di Matteo, T., Springel, V., \& Hernquist, L. 2005, Nature, 433, 604

Erb, D. 2015, Nature, 523, 169

Fabian, A. 2012, ARA\&A, 50, 455

Feltre, A., Bacon, R., Tresse, L., et al. 2018, A\&A, 617, A62

Feruglio, C., Maiolino, R., Piconcelli, E., et al. 2010, A\&A, 518, L155

Feruglio, C., Fiore, F., Carniani, S., et al. 2015, A\&A, 583, A99

Finley, H., Bouché, N., Contini, T., et al. 2017, A\&A, 608, A7

Fluetsch, A., Maiolino, R., Carniani, S., et al. 2018, MNRAS, submitted [arXiv:1805.05352]

Förster Schreiber, N. M., Übler, H., Davies, R. L., et al. 2018, ApJ, submitted [arXiv:1807.04738]

Fraternali, F. 2017, Astrophys. Space Sci. Lib., 430, 323

Fraternali, F., \& Binney, J. J. 2006, MNRAS, 366, 449

Genzel, R., Förster Schreiber, N. M., Rosario, D., et al. 2014, ApJ, 796, 7

Greene, J., \& Ho, L. 2005, ApJ, 627, 721

Guo, Q., White, S., Li, C., \& Boylan-Kolchin, M. 2010, MNRAS, 404, 111

Harrison, C., Alexander, D. M., Mullaney, J. R., \& Swinbank, A. M. 2014, MNRAS, 441, 3306

Harrison, C. M., Alexander, D. M., Mullaney, J. R., et al. 2016, MNRAS, 456, 1195

Heckman, T., Armus, L., \& Miley, G. 1990, ApJS, 74, 833

Heckman, T. M., Lehnert, M. D., Strickland, D. K., \& Armus, L. 2000, ApJS, 129,493
Henriques, B. M. B., White, S. D. M., Thomas, P. A., et al. 2017, MNRAS, 469, 2626

Hill, M. J., \& Zakamska, N. 2014, MNRAS, 439, 2701

Hopkins, P. F., Hernquist, L., Cox, T. J., et al. 2006, ApJS, 163, 50

Hopkins, P. F., Kereš, D., Oñorbe, J., et al. 2014, MNRAS, 445, 581

Jeong, H., Yi, S. K., Kyeong, J., et al. 2013, ApJS, 208, 7

Kauffmann, G., Heckman, T. M., White, S. D. M., et al. 2003, MNRAS, 341, 54

Kewley, L., Groves, B., Kauffmann, G., \& Heckman, T. 2006, MNRAS, 372, 961

King, A., \& Pounds, K. 2015, ARA\&A, 53, 115

Liddle, A. R. 2007, MNRAS, 377, L74

Madau, P., Ferguson, H. C., Dickinson, M., et al. 1996, MNRAS, 283, 1388

Maiolino, R., Gallerani, S., Neri, R., et al. 2012, MNRAS, 425, L66

Maiolino, R., Russell, H. R., Fabian, A. C., et al. 2017, Nature, 544, 202

Marasco, A., Fraternali, F., \& Binney, J. J. 2012, MNRAS, 419, 1107

Martin, C. 2005, ApJ, 621, 227

Martin, C. 2006, ApJ, 647, 222

Martin, C. L., Shapley, A. E., Coil, A. L., et al. 2012a, ApJ, 760, 127

Martin, C. L., Shapley, A. E., Coil, A. L., et al. 2012b, ApJ, 760, 127

Moster, B. P., Somerville, R., Maulbetsch, C., et al. 2010, ApJ, 710, 903

Moster, B. P., Naab, T., \& White, S. 2013, MNRAS, 428, 3121

Mullaney, J. R., Alexander, D. M., Fine, S., et al. 2013, MNRAS, 433, 622

Murray, N., Quataert, E., \& Thompson, T. A. 2005, ApJ, 618, 569

O'Connell, R. W. 1976, ApJ, 206, 370

Parikh, T., Thomas, D., Maraston, C., et al. 2018, MNRAS, 477, 3954

Perna, M., Brusa, M., Salvato, M., et al. 2015, A\&A, 583, A72

Perna, M., Lanzuisi, G., Brusa, M., Cresci, G., \& Mignoli, M. 2017a, A\&A, 606, A96

Perna, M., Lanzuisi, G., Brusa, M., Mignoli, M., \& Cresci, G. 2017b, A\&A, 603, A99

Peterson, R. C. 1976, ApJ, 210, L123

Pettini, M., Steidel, C., Adelberger, K., Dickinson, M., \& Giovalisco, M. 2000, ApJ, 528, 96

Prochaska, J. X., Kasen, D., \& Rubin, K. 2011, ApJ, 734, 24

Renzini, A., \& Peng, Y.-J. 2015, ApJ, 801, L29

Rodríguez Zaurín, J., Tadhunter, C. N., Rose, M., \& Holt, J. 2013, MNRAS, 432, 138

Rubin, K. H. R., Prochaska, J. X., Koo, D. C., et al. 2014, ApJ, 794, 156

Rupke, D. S., \& Veilleux, S. 2005, ApJ, 631, L37

Rupke, D., \& Veilleux, S. 2011, ApJ, 729, L27

Rupke, D., \& Veilleux, S. 2013a, ApJ, 768, 75

Rupke, D., \& Veilleux, S. 2013b, ApJ, 775, L15

Rupke, D. S. N., \& Veilleux, S. 2015, ApJ, 801, 126

Rupke, D., Veilleux, S., \& Sanders, D. 2002, ApJ, 570, 588

Rupke, D., Veilleux, S., \& Sanders, D. 2005a, ApJ, 160, 87

Rupke, D., Veilleux, S., \& Sanders, D. 2005b, ApJ, 160, 115

Rupke, D., Veilleux, S., \& Sanders, D. 2005c, ApJ, 632, 751

Rupke, D., Gültekin, K., \& Veilleux, S. 2017, ApJ, 850, 40

Salim, S., Rich, R. M., Charlot, S., et al. 2007, ApJS, 173, 267

Sarzi, M., Falcón-Barroso, J., Davies, R. L., et al. 2006, MNRAS, 366, 1151

Scarlata, C., \& Panagia, N. 2015, ApJ, 801, 43

Schawinski, K., Urry, C. M., Virani, S., et al. 2010, ApJ, 711, 284

Simard, L., Mendel, J. T., Patton, D. R., Ellison, S. L., \& McConnachie, A. W. 2011, ApJS, 196, 11

Spiniello, C., Trager, S. C., Koopmans, L. V. E., \& Chen, Y. P. 2012, ApJ, 753, L32

Spitzer, Jr., L. 1978, JRASC, 72, 349

Stasinska, G., Fernandes, R. C., Mateus, A., et al. 2006, MNRAS, 371, 972

Steidel, C. C., Erb, D. K., Shapley, A. E., et al. 2010, ApJ, 717, 289

Strauss, M. A., Weinberg, D. H., Lupton, R. H., et al. 2002, AJ, 124, 1810

Thomas, D., Maraston, C., \& Bender, R. 2003, MNRAS, 343, 279

Tremonti, C. A., Heckman, T. M., Kauffmann, G., et al. 2004, ApJ, 613, 898 van Dokkum, P. G., \& Conroy, C. 2010, Nature, 468, 940

van Dokkum, P. G., \& Conroy, C. 2012, ApJ, 760, 70

Veilleux, S., Cecil, G., \& Bland-Hawthorn, J. 2005, ARA\&A, 43, 769

Venturi, G., Nardini, E., Marconi, A., et al. 2018, A\&A, 619, A74

Villar-Martín, M., Humphrey, A., Delgado, R. G., Colina, L., \& Arribas, S. 2011, MNRAS, 418, 2032

Villar Martín, M., Emonts, B., Humphrey, A., Cabrera Lavers, A., \& Binette, L. 2014, MNRAS, 440, 3202

Weiner, B. J., Coil, A. L., Prochaska, J. X., et al. 2009, ApJ, 692, 187 Worthey, G. 1998, PASP, 110, 888

Worthey, G., Faber, S. M., Gonzalez, J. J., \& Burstein, D. 1994, ApJS, 94, 687

Worthey, G., Ingermann, B. A., \& Serven, J. 2011, ApJ, 729, 148

Yang, X., Mo, H. J., van den Bosch, F. C., et al. 2007, ApJ, 671, 153

York, D. G., Adelman, J., Anderson, Jr., J. E., et al. 2000, AJ, 120, 1579

Zakamska, N. L., Hamann, F., Pâris, I., et al. 2016, MNRAS, 459, 3144 